\title{
To Problem of the Rewinding of the Tape with Automatically Adjustable Influences
}

\author{
Muhsin Kh. Teshaev \\ Bukhara Engineering-Technological Institute, Bukhara, Republic of Uzbekistan \\ Email: muhsin_5@mail.ru, muhsin.teshayev@rambler.ru
}

Received 27 May 2014; revised 3 July 2014; accepted 16 July 2014

Copyright @ 2014 by author and Scientific Research Publishing Inc.

This work is licensed under the Creative Commons Attribution International License (CC BY).

http://creativecommons.org/licenses/by/4.0/

(c) (i) Open Access

\begin{abstract}
In this work the problem of rewinding of a tape with constant speed is considered. Considering that drums represent bodies of variable weight, the equations of motion of system are formulated. Taking into account parametrical clearing of system of servo-constraints, the structure of force of reaction of servo-constraints which provides steady realization of servo-constraints (a constancy of linear speed of a tape) is defined. For realization of servo-constraints, it is offered to build digital watching system (DWS) and the full system of equations of DWS is formed. Laws of change of the operating influences, systems providing stability under the relation of the variety defined of servo-constraints are defined.
\end{abstract}

\section{Keywords}

Rewinding of Tape, Servo-Constraint, Speed, Force of Reaction of Servo-Constraints, Parametrical Clearing, Stability, The Digital Watching System, Full System of the Equations

\section{Introduction}

For the first time the concept idea of servo-constraints has been entered into analytical dynamics by H. Beghin [1]. The methods used by H. Beghin, had the further development in P. Appel's [2] works, A. Przeborski's [3], V. S. Novoselov's [4], M. F. Shulgin's [5], V. V. Rumjantsev's [6] [7], V. I. Kirgetov’s [8], A. G. Azizov's [9] [10] and others.

Appendices of methods of analytical dynamics to a wide range of specific targets demand the account and other features connected with steady realization of servo-constraints, and that for such systems it is impossible to distract from a way of their realization.

S. S. Nugmanova's attention for the first time has been paid to this circumstance [11]. Following the theory of parametrical clearing [12], and the theory of the compelled motions [13] constructed the theory, allowing to de- 
velop the area of practical applicability of methods of analytical mechanics of systems with servo-constraints, including questions of their steady realization [14] [15]. In works [16] [17] the equations of motion of the systems, interfered by constraints of the first and second sort are deduced, and also the obvious kind of forces of reactions of servo-constraints is defined.

In this article the results of works [16] [17] are illustrated to the problem of rewinding of a tape.

\section{Forming the Equation of Motion and Refining the Servo-Constraints' Forces of Reaction}

Let's consider process of rewinding of a tape (Figure 1). Rewinding of a tape from the drum 3 on a drum 2 is carried out by means of electric machine (EM) of a direct current of the independent excitation operating through a reducer with transfer number $i_{1}$ on a drum 2. On Figure 1 the feedback providing a constancy of linear speed of motion of tape $v=$ const and forming of stabilizing pressure on an input of the electric machine (EM) are illustrated. As well as, from the work of Zijatdinov R. M. (1983) [18], we will divide the scheme Figure 1 on two parts on a dashed line. Kinetic energy of system looks like as:

$$
T=\frac{1}{2}\left[J_{2}\left(\varphi_{2}\right) \varphi_{2}^{2}+J_{1} \cdot \dot{\varphi}_{1}^{2}+J_{3}\left(\varphi_{3}\right) \dot{\varphi}_{3}^{2}\right]
$$

where $J_{1}, J_{2}, J_{3}$-the moments of inertia of a reducer both corresponding drums; $\varphi_{2}, \varphi_{3}$-angles of rotation of drums 2 and 3.

As drums 2 and 3 represent bodies of variable weight it is necessary to take the general equation of dynamics in the form showed in Bessonov A. P. (1967) [19]:

$$
\sum_{i=1}^{3}\left(\frac{\mathrm{d}^{*}}{\mathrm{~d} t} \frac{\partial^{*} T}{\partial \dot{q}_{i}}-\frac{\partial^{*} T}{\partial q_{i}}\right) \delta q_{i}=\sum_{i=1}^{3}\left(Q_{i}+R_{i}\right) \delta q_{i}
$$

where for $q_{i}$ co-ordinates $\varphi_{1}, \varphi_{2}, \varphi_{3}$ are designated; $Q_{i}$ 一the generalized force appropriated to co-ordinate $q_{i} ; R_{i}$-the generalized reactive force, which looks like

$$
R_{i}=\sum_{v=1}^{n}\left[\frac{\mathrm{d} m_{v}}{\mathrm{~d} t}\left(\bar{U}_{v}-\bar{v}_{v}\right)-m_{v} \bar{a}_{v}^{r}-2\left(\vec{\omega} \times \bar{v}_{r}\right)\right] \frac{\mathrm{d} \bar{r}_{v}}{\mathrm{~d} q_{i}}
$$

here $\left(\bar{U}_{v}-v_{v}\right)$-relative speed of joining particles; $\bar{a}_{v}^{r}$-particle acceleration in motion concerning the system of co-ordinates connected by a link, and the symbol “*” above specifies that differentiation is made according to a hardening principle.

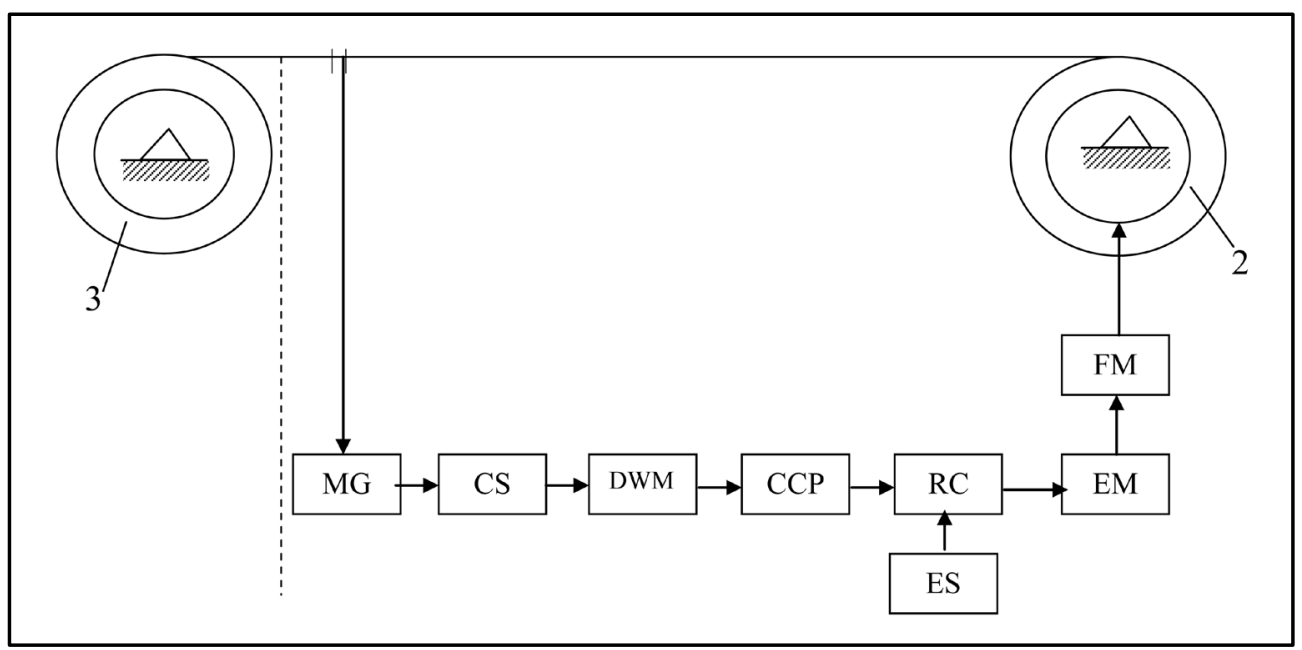

FM-forwarding mechanism; EM-electric machine; ES-energy source; RC-rein forcer-changer; CCP-converter of code in pressure; CS—converter scheme; DWM—digital watching machine; MG—-measuring gauge.

Figure 1. Process of rewinding of a tape. 
If relative speed of joining particles is equal to zero, and there is no relative motion of joining particles concerning a drum 2, according to Bessonov A. P. [16] (1967) we conclude that:

$$
R_{i}=0
$$

On system, according to assumptions, one ideal geometrical constraint

$$
\frac{\varphi_{1}}{\varphi_{2}}=i_{1}
$$

and one kinematical servo-constraint in Beghin H. (1967) [1]

$$
\left(\rho_{20}+h \frac{\varphi_{2}}{2 \pi}\right) \dot{\varphi}_{2}=v_{0}
$$

is imposed, where $\rho_{20}$-radius of an empty drum 2; $h$-a thickness of a tape.

Taking into account (3) kinetic energy (1) systems we will lead to a formula

$$
T=\frac{1}{2}\left[J_{2}\left(\varphi_{2}\right)+J_{1} \cdot i_{1}^{2}\right] \dot{\varphi}_{2}^{2}+\frac{1}{2} J_{3}\left(\varphi_{3}\right) \cdot \dot{\varphi}_{3}^{2}
$$

On possible moving constraint (3) imposes restriction [7]:

$$
\delta \varphi_{1}=i_{1} \delta \varphi_{2}
$$

From a way of action of servo-constraint (4) follows that, moving, on which servo-constraint works do not make reaction, look like in Beghin H. (1967) [1]:

$$
\delta \varphi_{1}=0
$$

We will consider the right part from a dashed line. Considering that for the right part,

$$
\sum_{\alpha_{3}=1}^{2} Q_{\alpha_{3}} \delta \varphi_{\alpha_{3}}=\left[M_{\ni л} \cdot i_{1}^{2}-F\left(\rho_{20}+h \frac{\varphi_{2}}{2 \pi}\right)\right] \delta \varphi_{2}
$$

where $M_{э n}$-the constant moment put EM under the influence of direct current; $F$ - force of a tension of a tape, from (2), (5)-(8), by method (A)-moving [6] [7], we will receive:

$$
\left[J_{1} \cdot \dot{i}_{1}^{2} \cdot J_{2}\left(\varphi_{2}\right)\right] \ddot{\varphi}_{2}=M_{9 \cdot n} \cdot i_{1}-F\left(\rho_{20}+h \frac{\varphi_{2}}{2 \pi}\right)+i \lambda
$$

where $\lambda$-reaction of servo-constraint (4).

Considering that for the left part

$$
Q_{3}=F\left(\rho_{3}-h \frac{\varphi_{3}}{2 \pi}\right)-M_{m p}
$$

where $\rho_{3}$-radius of no to wind off drum 3; $M_{m p}$-the brake moment of a drum 3, from (5), (10) we will receive

$$
J_{3}\left(\varphi_{3}\right) \ddot{\varphi}_{3}=F\left(\rho_{3}-h \frac{\varphi_{3}}{2 \pi}\right)-M_{m p}
$$

To the received system of the Equations (9), (11) adding one kinematical equation,

$$
\left(\rho_{3}-h \frac{\varphi_{3}}{2 \pi}\right) \dot{\varphi}_{3}=\left(\rho_{20}+h \frac{\varphi_{2}}{2 \pi}\right) \dot{\varphi}_{2}
$$

will be received system of three Equations (9), (11), (12) concerning unknown persons $\varphi_{2}, \varphi_{3}, \lambda, F, M_{m p}$.

Consider a case, when rewinding is carried out with constant brake moment $M_{m p}=$ const, and speed of a tape $v$ is regulated only.

As it is known [9] [10], servo-constraints are carried out not precisely and therefore, along with (4) the have occurrence parity 


$$
\left(\rho_{20}+h \frac{\varphi_{2}}{2 \pi}\right) \varphi_{2}-v_{o}=\xi
$$

where $\xi$-the parameter, characterizing clearing of system from servo-constraint (4).

Having for an object steady realization of servo-constraint (4), the received system of the equations will be added to (9), (11), (12) equation [9] [10]:

$$
\dot{\xi}=\tilde{U}
$$

and reaction compulsion $\tilde{U}$ we set in a kind,

$$
\tilde{U}=-\kappa_{3} \xi
$$

where $\kappa_{3}$-a positive constant at the expense of which choice there is a possibility to satisfy to quality of transient [19] [20]. Taking into account (3), (15), Equation (14) we will lead to the following:

$$
\left(\rho_{20}+h \frac{\varphi_{2}}{2 \pi}\right) \ddot{\varphi}_{2}+h \frac{\dot{\varphi}_{2}^{2}}{2 \pi}+\kappa_{5}\left(\rho_{20}+h \frac{\varphi_{2}}{2 \pi}\right) \dot{\varphi}_{2}-\kappa_{5} v_{6}=0
$$

Thus, the system of the Equations (9), (11), (12), (16) describes dynamics of adjustable process of rewinding of a tape concerning variables $\varphi_{2}, \dot{\varphi}_{2}, F, \lambda$. From system of the Equations (9), (11), (12), (16) can be defined $\lambda$ as function of variables $\varphi_{2}, \dot{\varphi}_{2}$, and by that reaction of servo-constraint in the form of the feedback law. For this purpose from the Equation (11) we will define force $F$ :

$$
F=\frac{1}{\rho_{3}-h \frac{\varphi_{2}}{2 \pi}}\left[J_{3}\left(\varphi_{3}\right) \ddot{\varphi}_{3}+M_{m p}\right]
$$

and substituting it in (9), the following equation will be received:

$$
\left[J_{1} \cdot \dot{i}_{1}^{2}+J_{2}\left(\varphi_{2}\right)\right] \ddot{\varphi}_{2}=M_{э л} \cdot i_{1}-\frac{\rho_{20}+h\left(\varphi_{2} / 2 \pi\right)}{\rho_{3}-h\left(\varphi_{3} / 2 \pi\right)} J_{3}\left(\varphi_{3}\right) \ddot{\varphi}_{3}+M_{m p}+i_{1} \lambda .
$$

Taking into account (12), Equation (17) can be led to the following:

$$
\begin{aligned}
& {\left[J_{1} \cdot \dot{1}_{1}^{2}+J_{2}\left(\varphi_{2}\right)\right] \cdot \dot{\varphi}_{2} \ddot{\varphi}_{2}\left(\rho_{20}+h \frac{\varphi_{2}}{2 \pi}\right)-M_{э \pi} \cdot \dot{i}_{1}\left(\rho_{20}+h \frac{\varphi_{2}}{2 \pi}\right) \cdot \dot{\varphi}_{2}} \\
& +J_{3}\left(\varphi_{3}\right)\left(\rho_{3}-h \frac{\varphi_{3}}{2 \pi}\right) \dot{\varphi}_{3} \ddot{\varphi}_{3}+M_{m p}\left(\rho_{3}-h \frac{\varphi_{3}}{2 \pi}\right) \cdot \dot{\varphi}_{3} \\
& -i_{1} \cdot \lambda\left(\rho_{20}+h \frac{\varphi_{2}}{2 \pi}\right) \dot{\varphi}_{2}=0
\end{aligned}
$$

\section{Stability}

If a reaction of servo-constraint $\lambda$ to form under the law, showed in [17] [18]:

$$
\lambda=-\frac{1}{i_{1}}\left\{\frac{J_{1} \cdot \dot{i}_{1}^{2}+J_{2}\left(\varphi_{2}\right)}{\rho_{20}+h\left(\varphi_{2} / 2 \pi\right)} \frac{\dot{\varphi}_{2}^{3}}{2 \pi}-e_{1}\right\}
$$

where $e_{1}$-a positive constant, the Equation (18) is led to a kind:

$$
\begin{aligned}
& {\left[J_{1} \cdot \dot{1}_{1}^{2}+J_{2}\left(\varphi_{2}\right)\right] \dot{\varphi}_{2} \ddot{\varphi}_{2}\left(\rho_{20}+h \frac{\varphi_{2}}{2 \pi}\right)} \\
& +\left[J_{1} \cdot \dot{i}_{1}^{2}+J_{2}\left(\varphi_{2}\right)\right] \cdot h \frac{\dot{\varphi}_{2}^{3}}{2 \pi} \\
& +\left[J_{3}\left(\varphi_{3}\right)+M_{m p}-M_{\ni \pi} \cdot \dot{i}_{1}+e_{1}\right]\left(\rho_{20}+h \frac{\varphi_{2}}{2 \pi}\right) \dot{\varphi}_{2}=0
\end{aligned}
$$


Taking into account a parity (13), last equation will look like:

$$
\left(J_{1} \cdot \dot{i}_{1}^{2}+J_{2}\left(\varphi_{2}\right)\right) \dot{\varphi}_{2} \dot{\xi}+\left[J_{3}\left(\varphi_{3}\right)+M_{m p}-M_{э л} \cdot \dot{1}_{1}+e_{1}\right]\left(v_{o}+\xi\right)=0
$$

Considering $\xi=0, \dot{\xi}=0$, from (19) we will receive

$$
\left(J_{3}\left(\varphi_{3}\right)+M_{m p}-M_{9,2} \cdot i_{1}+e_{1}\right) \cdot v_{o}=0
$$

Taking into account (20), Equation (19) will look like

$$
\dot{\xi}+m_{1} \xi=0
$$

where

$$
m_{1}=\frac{J_{3}\left(\varphi_{3}\right)+M_{m p}-M_{э n} \cdot i_{1}+e_{1}}{J_{1} \cdot \dot{i}_{1}^{2}+J_{2}\left(\varphi_{2}\right)}
$$

Apparently, from (22) the Equation (21) represents the differential equation with variable factors. Stability of its zero decision $\xi=0$ it is going to be investigated by a method of functions of Lyapunov, developed for unsteady systems [16] [17]. The auxiliary equation to (21) will look like:

$$
\dot{\xi}+m_{1}^{o} \xi=0
$$

where

$$
m_{1}^{o}=\frac{1}{J_{1} \cdot \dot{i}_{1}^{2}+J_{20}\left(\varphi_{20}\right)} \cdot\left[J_{30}\left(\varphi_{30}\right)+M_{m p}-M_{э n} \cdot i_{1}+e_{1}\right]
$$

In expression (24) constant number $e_{1}$ we will choose such, that a root $\lambda$ of the characteristic Equation (23)

$$
\lambda+m_{1}^{o}=0
$$

had a negative material part. This condition, according to Hurvits’s criterion [21], will look like

$$
m_{1}^{o}>0
$$

which is reduced to a condition

$$
e_{1}>M_{э л} \cdot \dot{i}_{1}-M_{m p}-J_{30}\left(\varphi_{30}\right)
$$

Lyapunov's definitely positive function $v(\xi)$ will be chosen as:

$$
v(\xi)=\frac{1}{2} \xi^{2} .
$$

Its full derivative on time, worked out owing to the equation of the indignant motion (23), will look like:

$$
\left(\frac{\mathrm{d} v}{\mathrm{~d} t}\right)_{23}=w(\xi)=-m_{1} \xi^{2}
$$

Let's calculate: $\left(\frac{\mathrm{d} v}{\mathrm{~d} t}\right)_{21}$

$$
\left(\frac{\mathrm{d} v}{\mathrm{~d} t}\right)_{21}=w(\xi)+\left(-m_{1}+m_{1}^{o}\right) \xi^{2}=-m_{1} \xi^{2} .
$$

Then the condition of certain positively of the form- $\left(\frac{\mathrm{d} v}{\mathrm{~d} t}\right)_{21}$ will look like

$$
m_{1}>\gamma_{2}, \quad\left(\gamma_{2}>0\right)
$$

which it is reduced to a condition 


$$
J_{3}\left(\varphi_{3}\right)<M_{э, n} \cdot i_{1}-M_{m p}-\gamma_{2}\left[J_{1} \cdot \dot{i}_{1}^{2}+J_{2}\left(\varphi_{2}\right)\right]
$$

Condition (26) show that, for maintenance of steady realization of servo-constraint (4) moment of the electric machine $M_{э n}(\mathrm{EM})$, the moment of friction $M_{m p}$, the moments of inertia $J_{1}, J_{2}, J_{3}$ and a positive constant $\gamma_{2}$ it is necessary to choose on corresponding condition.

\section{The Realization of Servo-Constraints}

We will consider a realization problem of servo-constraint (4) by electromechanical digital watching system (DWS) [20] for which executive element we accept the engine of a direct current of independent excitation. Its full system of the equations will look like [17]:

$$
\begin{gathered}
{\left[J_{1} \cdot i_{1}^{2}+J_{2}\left(\varphi_{2}\right)\right] \dot{\varphi}_{2} \ddot{\varphi}_{2}\left(\rho_{20}+h \frac{\varphi_{2}}{2 \pi}\right)} \\
-M_{э \pi} \cdot i_{1}\left(\rho_{20}+h \frac{\varphi_{2}}{2 \pi}\right) \dot{\varphi}_{2}+J_{2}\left(\varphi_{3}\right) \cdot\left(\rho_{3}-h \frac{\varphi_{3}}{2 \pi}\right) \dot{\varphi}_{3} \ddot{\varphi}_{3} \\
+M_{m p}\left(\rho_{3}-h \frac{\varphi_{3}}{2 \pi}\right) \dot{\varphi}_{3}+J_{g} \cdot i_{1}^{2} \cdot \ddot{\varphi}_{3} \cdot\left(\rho_{20}+h \frac{\varphi_{2}}{2 \pi}\right) \dot{\varphi}_{2} \\
-i_{1} K_{m_{1}} \cdot I_{1} J_{я}=0 \\
L_{1} \dot{I}_{1}+R_{1} I_{1}+K_{w_{1}} \cdot \dot{i}_{1} \cdot \dot{\varphi}_{2}=U^{\mathrm{RC}}, \\
T^{\mathrm{RC}} \cdot \dot{U}^{\mathrm{RC}}+U^{\mathrm{RC}}=K^{\mathrm{RC}} \cdot U^{\mathrm{CCP}}, \\
T^{\mathrm{CCP}} \cdot \dot{U}^{\mathrm{CCP}}+U^{\mathrm{CCP}}=K^{\mathrm{CCP}} \cdot x^{\mathrm{DWM}}, \\
T^{\mathrm{DWM}} \cdot \dot{x}^{\mathrm{DWM}}+x^{\mathrm{DWM}}=f\left(\varphi_{2}, \dot{\varphi}_{2}, L_{1}, T^{\mathrm{RC}}, T^{\mathrm{CCP}}, \cdots\right), \\
T_{\alpha_{3}}^{\mathrm{CS}} \dot{x}_{\alpha_{3}}^{\mathrm{CS}}+x_{\alpha_{3}}^{\mathrm{CS}}=K_{\alpha_{3}}^{\mathrm{CS}} U_{\alpha_{3}}^{\mathrm{CS}}, \quad\left(a_{3}=1, \cdots, 2\right) \\
T_{1}^{\mathrm{D}} \dot{U}_{1}^{\mathrm{MG}}+U_{1}^{\mathrm{MG}}=K_{1}^{\mathrm{MG}} \varphi_{2}, \quad T_{2}^{\mathrm{MG}} \dot{U}_{2}^{\mathrm{MG}}+U_{2}^{\mathrm{MG}}=K_{2}^{\mathrm{MG}} \dot{\varphi}_{2}
\end{gathered}
$$

where $L_{i}, R_{i}$-inductive and ohmic resistance EM; $T^{\mathrm{RC}}, T^{\mathrm{CCP}}, T^{\mathrm{DWM}}, T^{\mathrm{CS}}, T^{D}, T^{\mathrm{MG}}$-delay factors; $K^{\mathrm{RC}}$, $K^{\mathrm{CCP}}, T K^{\mathrm{CS}}, K^{D}, K^{\mathrm{MG}}$ - strengthening factors; $K_{w}, K_{m}$-factor against-EDS and the rotary moment; $U^{\mathrm{RC}}-$ entrance pressure EM; $U^{\mathrm{CCP}}$-target pressure of the converter of code in pressure (CCP); $x^{\mathrm{DWM}}$-target parameter DWM; $U_{1}^{\mathrm{MG}}, U_{2}^{\mathrm{MG}}, x_{\alpha_{3}}^{\mathrm{CS}}$-target parameters of gauges measurements (MG) and schemes of transformations (converter scheme) (CS); the moment of inertia of anchor of EM;

$f\left(\varphi_{2}, \dot{\varphi}_{2}, L_{1}, T^{\mathrm{RC}}, T^{\mathrm{CCP}}, \cdots\right)$ -

The law of formation of the operating influences DWM providing stability of motion of system in relation to servo-constraint (4) is defined by the decision of system of the equations:

$$
\begin{aligned}
& -\frac{1}{i_{1}}\left\{\frac{J_{1} \cdot i_{1}^{2}+J_{2}\left(\varphi_{2}\right)}{\left(\rho_{20}+h(\varphi)\right) \dot{\varphi}_{2}} \cdot \frac{\dot{\varphi}_{2}^{3}}{2 \pi}-e_{1}\right\}+J_{я} \cdot i_{1}^{2} \ddot{\varphi}_{2}-i_{1} \cdot K_{m_{1}} I_{1}=0 \\
& L_{1} \dot{I}_{1}+R_{1} I_{1}+K_{w_{1}} \cdot \dot{i}_{1} \cdot \dot{\varphi}_{2}=U^{\mathrm{RC}}, \\
& T^{\mathrm{RC}} \cdot \dot{U}^{\mathrm{RC}}+U^{\mathrm{RC}}=K^{\mathrm{RC}} \cdot U^{\mathrm{CCP}}, \\
& T^{\mathrm{CCP}} \cdot \dot{U}^{\mathrm{CCP}}+U^{\mathrm{CCP}}=K^{\mathrm{CCP}} \cdot x^{\mathrm{DWM}}, \\
& T^{\mathrm{DWM}} \cdot \dot{x}^{\mathrm{DWM}}+x^{\mathrm{DWM}}=f\left(\varphi_{2}, \dot{\varphi}_{2}, L_{1}, T^{\mathrm{RC}}, T^{\mathrm{CCP}}, \cdots\right) \text {, } \\
& T_{\alpha_{3}}^{\mathrm{CS}} \dot{x}_{\alpha_{3}}^{\mathrm{CS}}+x_{\alpha_{3}}^{\mathrm{CS}}=K_{\alpha_{3}}^{\mathrm{CS}} U_{\alpha_{3}}^{\mathrm{CS}}, \quad\left(a_{3}=1, \cdots, 2\right) \\
& T_{1}^{\mathrm{MG}} \dot{U}_{1}^{\mathrm{MG}}+U_{1}^{\mathrm{MG}}=K_{1}^{\mathrm{MG}} \varphi_{2}, \quad T_{2}^{\mathrm{MG}} \dot{U}_{2}^{\mathrm{MG}}+U_{2}^{\mathrm{MG}}=K_{2}^{\mathrm{MG}} \dot{\varphi}_{2}
\end{aligned}
$$

rather of $f\left(\varphi_{2}, \dot{\varphi}_{2}, L_{1}, T^{\mathrm{RC}}, T^{\mathrm{CCP}}, \cdots\right)$. This law looks like: 


$$
\begin{aligned}
& f=\frac{T^{\mathrm{DWM}} \cdot T^{\mathrm{CCP}} \cdot T^{\mathrm{RC}} \cdot L_{1}}{i_{1} \cdot K_{1}^{\mathrm{RC}} \cdot K_{1}^{\mathrm{CCP}} \cdot K_{m_{1}}}\left\{-\frac{1}{i_{1}}\left[\frac{J_{1} \cdot \dot{i}_{1}^{2}+J_{2}\left(\varphi_{2}\right)}{\rho_{20}+h\left(\varphi_{2} / 2 \pi\right)} \cdot \frac{\dot{\varphi}_{2}^{2}}{2 \pi}\right]+J_{g} \cdot \dot{i}_{1}^{2} \cdot \ddot{\varphi}_{2}\right\}^{(I V)} \\
& +\frac{T^{\mathrm{CCP}}\left(L_{1}+T^{\mathrm{RC}} \cdot R_{1}+T^{\mathrm{RC}} \cdot L_{1}\right)+L_{1} \cdot T^{\mathrm{RC}}}{i_{1} \cdot K_{1}^{\mathrm{RC}} \cdot K_{1}^{\mathrm{CCP}} \cdot K_{m_{1}}} \cdot\left\{-\frac{1}{i_{1}}\left[\frac{J_{1} \cdot \dot{i}_{1}^{2}+J_{2}\left(\varphi_{2}\right)}{\rho_{20}+h\left(\varphi_{2} / 2 \pi\right)} \cdot \frac{\dot{\varphi}_{2}^{2}}{2 \pi}\right]+J_{\Omega} \cdot \dot{i}_{1}^{2} \cdot \ddot{\varphi}_{2}\right\}^{\prime \prime \prime} \\
& +\frac{T^{\mathrm{DWM}}\left(L_{1} \cdot\left(1+T^{\mathrm{RC}}\right)+T^{\mathrm{CCP}} \cdot R_{1}\right)+T^{\mathrm{CCP}}\left(L_{1}+R_{1} \cdot T^{\mathrm{RC}}\right)+L_{1} \cdot T^{\mathrm{RC}}}{i_{1} \cdot K_{1}^{\mathrm{RC}} \cdot K_{1}^{\mathrm{CCP}} \cdot K_{m_{1}}} \\
& \cdot\left\{-\frac{1}{i_{1}}\left[\frac{J_{1} \cdot \dot{i}_{1}^{2}+J_{2}\left(\varphi_{2}\right)}{\rho_{20}+h\left(\varphi_{2} / 2 \pi\right)} \cdot \frac{\dot{\varphi}_{2}^{2}}{2 \pi}\right]+J_{g} \cdot i_{1}^{2} \cdot \ddot{\varphi}_{2}\right\}^{\prime \prime}+\frac{L\left(1+T^{\mathrm{RC}}\right)+R_{1}\left(T^{\mathrm{DWM}}+T^{\mathrm{CCP}}\right)}{i_{1} \cdot K_{1}^{\mathrm{RC}} \cdot K_{1}^{\mathrm{CCP}} \cdot K_{m_{1}}} \\
& \cdot\left\{-\frac{1}{i_{1}}\left[\frac{J_{1} \cdot \dot{i}_{1}^{2}+J_{2}\left(\varphi_{2}\right)}{\rho_{20}+h\left(\varphi_{2} / 2 \pi\right)} \cdot \frac{\dot{\varphi}_{2}^{2}}{2 \pi}\right]+J_{g} \cdot \dot{i}_{1}^{2} \cdot \ddot{\varphi}_{2}\right\}^{\prime}+\frac{T^{\mathrm{DWM}} \cdot T^{\mathrm{CCP}} \cdot T^{\mathrm{RC}} \cdot K_{w_{1}} \cdot i_{1}}{K_{1}^{\mathrm{RC}} \cdot K_{1}^{\mathrm{CCP}}} \cdot \varphi_{2}^{(I V)} \\
& +\frac{\left(T^{\mathrm{DWM}}\left(T^{\mathrm{RC}}+T^{\mathrm{CCP}}\right)+T^{\mathrm{RC}} \cdot T^{\mathrm{CCP}}\right) \cdot K_{w_{1}} \cdot i_{1}}{K_{1}^{\mathrm{RC}} \cdot K_{1}^{\mathrm{CCP}}} \cdot \dddot{\varphi}_{2} \\
& +\frac{\left[T^{\mathrm{DWM}}+T^{\mathrm{CCP}}+T^{\mathrm{RC}}\right] \cdot K_{w_{1}} \cdot \dot{1}_{1}}{K_{1}^{\mathrm{RC}} \cdot K_{1}^{\mathrm{CCP}}} \cdot \ddot{\varphi}_{2}+\frac{K_{w_{1}} \cdot \dot{i}_{1}}{K_{1}^{\mathrm{RC}} \cdot K_{1}^{\mathrm{CCP}}} \cdot \dot{\varphi}_{2}
\end{aligned}
$$

Substituting the law (29) in (27), results the Equation (21). Hence, the law (28) provides asymptotically stability of motion of system under the relation of the variety defined by servo-constraint (4).

Along with the generalized model, we will consider the simplified model of watching system [17] [18], i.e. at assumptions,

$$
L_{1}=T^{\mathrm{RC}}=T^{\mathrm{CCP}}=T^{\mathrm{DWM}}=0, T_{1}^{\mathrm{MG}}=T_{2}^{\mathrm{MG}}=0, \quad T_{\alpha_{3}}^{\mathrm{CS}}=0,\left(\alpha_{3}=1,2\right),
$$

from (28) we will receive the law of formation of operating influences DWM for simplified model DWS:

$$
x^{\mathrm{DWM}}=\frac{R_{1}}{i_{1} \cdot K_{1}^{\mathrm{RC}} \cdot K_{1}^{\mathrm{CCP}} \cdot K_{m_{1}}} \cdot\left\{-\frac{1}{i_{1}} \frac{J_{1} \cdot i_{1}^{2}+J_{2}\left(\varphi_{2}\right)}{\left(\rho_{20}+h \varphi_{2} / 2 \pi\right) \dot{\varphi}_{2}} \cdot \frac{\dot{\varphi}_{2}^{3}}{2 \pi}-e_{1}+J_{q} \cdot i_{1}^{2} \cdot \ddot{\varphi}_{2}\right\}+\frac{K_{w_{1}} \cdot i_{1} \cdot \dot{\varphi}_{2}}{K^{\mathrm{RC}} \cdot K^{\mathrm{CCP}}}
$$

Substituting the law (31) in (27) at the same assumptions (30) we will receive the Equation (21), which stability conditions looks like (26).

\section{References}

[1] Beghin, H. (1967) The Theory of Hygroscopic Compasses. Nauka Press, Moscow, 5-31.

[2] Appel, P. (1925) Sur les une forme generale des equations de la dynamique (memorial des Sciences Mathematique, fasccule 1). Paris, 1-50.

[3] Przeborski, A. (1902) Die allgemeinsten Gleichunden der Klassischen Dunamik. Mathematische Zeitschrift, 36, 184194. http://dx.doi.org/10.1007/BF01188619

[4] Novoselov, V.S. (1969) New Settlers Nonlinear Nonholonomic Co-Ordinates in the Analytical Mechanics. Leningrad State University, 217, 50-83.

[5] Shulgin, M.F. (1958) About Some Differential Equations of Analytical Dynamics and Their Integration. The Central Asian State University Press, Tashkent, 73-94.

[6] Rumyantsev, V.V. (1961) About Motion of Some Systems with Nonideal Constraints. The Moscow State University bulletin, Series, Mathematics and Mechanics, 61-66.

[7] Rumyantsev, V.V. (1976) About Motion of Operated Mechanical Systems. The Journal of Applied Mathematics and Mechanics, 5, 771-781.

[8] Kirgetov, V.I. (1967) About Motion of Operated Mechanical Systems with Conditional Constrains (Servo-Constraints). The Journal of Applied Mathematics and Mechanics, 3, 433-446. 
[9] Azizov, A.G. (1975) About the Equations of Dynamics of Systems with Servo-Constraints. Proceedings of Tashkent State University, 476, 67-75.

[10] Azizov, A.G. (1980) Applied Problems of Dynamics of Operated Systems. Tashkent State University, Tashkent, 1-28.

[11] Nugmanova, Sh.S. (1953) About the Equations of Motions of Adjustable Systems. The Kazan Aviation Institute, 27, 26-35.

[12] Chetaev, N.G. (1962) About a Principle of House. In: Chetaev, N.G., Ed., Stability of Motion. Works on the Analytical Mechanics, Academy of Science of the USSR, 329-334.

[13] Chetaev, N.G. (1962) On the Compelled Motions. In: Chetaev, N.G., Ed., Stability of Motion. Works on the Analytical Mechanics, Academy of Science of the USSR, 311-316.

[14] Krasovsky, N.N. (1959) Some of Problems of Theories of Stability of Motion. Fizmatgiz, Moscow, 17-139.

[15] Lure, A.I. (1961) Analytical Mechanics. Fizmatgiz, Moscow, 13-430.

[16] Teshaev, M.Kh. (1999) About Stabilization of the Mechanical Systems Constrained by Geometrical Constriets. The Uzbek Magazine "Problems of Mechanics", 1, 7-20.

[17] Teshaev, M.Kh. (2005) About Designing of Reactions of Servo-Constraints the Systems Constrained by Kinematical Constraints. The Uzbek Magazine "Problems of Mechanics", 1, 3-7.

[18] Ziyatdinov, R.M. (1980) Dynamic of Machine Units with Automatically Adjustable Variators. Tashkent, 1-23.

[19] Bessonov, A.P. (1967) Bas of Dynamics of Mechanisms with Variable Weight of Links. The Nauka, Moscow, 84-102.

[20] Medvedev, V.S. (1979) Designing of Watching Drives by Means of the COMPUTER. Mechanical Engineering, Moscow, 47-62.

[21] Merkin, G.D. (1987) Introduction to Theory of Stability. The Nauka, Moscow, 1-304. 
Scientific Research Publishing (SCIRP) is one of the largest Open Access journal publishers. It is currently publishing more than 200 open access, online, peer-reviewed journals covering a wide range of academic disciplines. SCIRP serves the worldwide academic communities and contributes to the progress and application of science with its publication.

Other selected journals from SCIRP are listed as below. Submit your manuscript to us via either submit@scirp.org or Online Submission Portal.
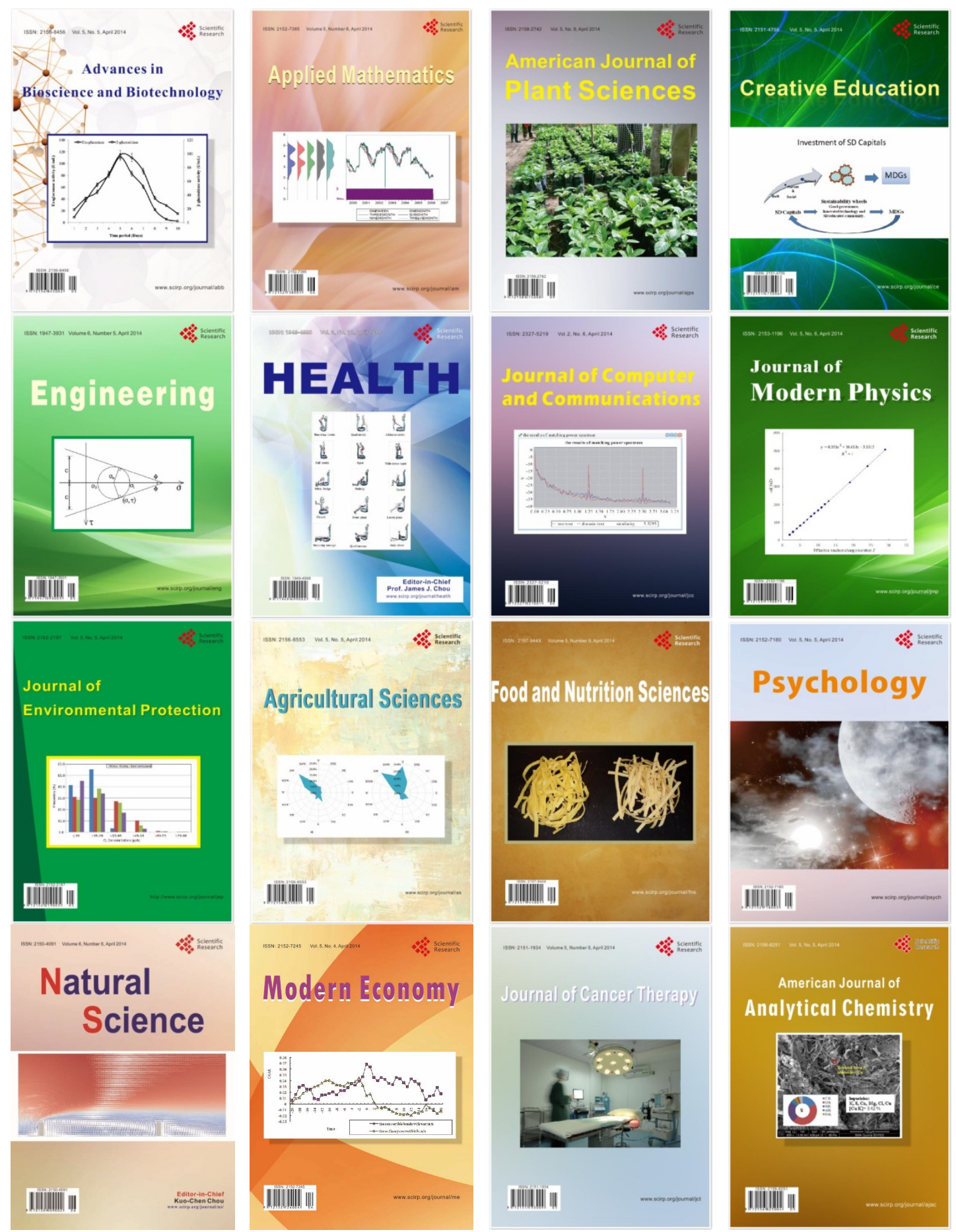